\title{
Animal Assisted Therapy in a Special Needs Dental Practice: An Interprofessional Model for Anxiety Reduction
}

\author{
Caren M. Cajares ${ }^{1, *}$, Carolyn M. Rutledge ${ }^{2}$ and Tina S. Haney ${ }^{2}$ \\ 131 Gallant Fox Road, Virginia Beach, VA 23462, USA \\ ${ }^{2}$ Old Dominion University, Health Science Building, 4608 Hampton Blvd., Norfolk, VA 23529, USA
}

\begin{abstract}
Purpose: Individuals with intellectual and developmental disabilities (IDD) suffer from poor oral hygiene and periodontal disease that may predispose them to systemic diseases. In order to receive dental care, the assistance of a certified registered nurse anesthetist (CRNA) is often needed. The role of the CRNA is to provide sedation to reduce the high level of anxiety demonstrated by many individuals with intellectual disabilities in the dental suite. However, this can become a challenge with patients that are anxious when they arrive. The purpose of this paper is to describe an interprofessional dental team that added a certified therapy dog and handler to reduce anxiety of individuals with IDD when they arrive in the dental suite.
\end{abstract}

Methods: A convenience sample of 30 individuals with intellectual disability seen for dental care in an outpatient setting met with a therapy dog prior to receiving preventative dental care. Comparisons were made between observed anxiety levels and behaviors measured by the ADAMS (anxiety scale) and a researcher-developed behavior tool prior to and after the interaction with the therapy dog.

Results: This program suggested that the addition of the therapy dog to the interprofessional team prior to sedation decreased anxiety levels and improved the behavioral outcomes of the individual with intellectual disabilities.

Conclusions: The incorporation of a certified therapy dog and handler as part of an interprofessional healthcare team in the dental suite may pay great dividends in improving the compliance and comfort of the individual with intellectual and developmental disabilities during dental care visits.

Keywords: Interprofessional practice, Healthcare teams, Collaboration, Intellectual Disability, Animal Assisted Therapy, Dental Care.

Dental care for individuals with intellectual and developmental disabilities (IDD) is extremely important in optimizing overall health. Yet, it can be challenging as a result of high levels of anxiety demonstrated by many of the individuals during a dental visit. Further, each individual with IDD comes from a different culture, is a different size and age, and may present with multiple medical, social, and psychological needs. Their response to care is all too often unpredictable [1]. Office approaches in treating individuals with IDD must be responsive to these differences. The ingenuity that is necessary in treating individuals with IDD in a dental practice can be based on sound scientific principles, but also are improved with tried and true alternative therapies. Individualized care for those with IDD requires a dedicated team approach where adjustments are made to accommodate the differing needs of this population of patients.

Approximately fifteen percent of children 3-7 years of age or 10 million children were identified as having a disability in 2007-2008 [2]. This number increased by $17 \%$ over less than a decade, and it is anticipated to

*Address correspondence to this author at the 131 Gallant Fox Road, Virginia Beach, VA 23462, USA; Tel: (757) 615-3572; Fax: (757) 819-4969;

E-mail: cccrna@icloud.com continue to climb [2]. People with IDD are more likely to have poor oral hygiene, periodontal disease, and untreated dental caries than members of the general population [3]. The Surgeon General reported in 2010 that oral diseases and disorders affect health and well being throughout life. The burden of oral problems is extensive and may be particularly severe in vulnerable populations [4]. Despite the use of anesthesia for dental treatments, the individual with intellectual disability often presents with symptoms of anxiety pre and post dental procedures $[5,6]$.

Dental practices are traditionally seen as possessing well-oiled teams who are trained in and focused on oral health. Most dental practice teams consist of a dentist, a dental hygienist, and dental assistant. In order to meet the needs of individuals with IDD, an expanded interprofessional team may incorporate a certified registered nurse anesthetist (CRNA) [7]. The CRNA typically provides anesthesia to the individual with intellectual disabilities in order to reduce their anxiety and improve cooperation with typical oral care. Together, the team is able to meet most of the oral care needs of the individual with IDD.

Those with a diagnosis of IDD are often anxious or distressed when visiting health care providers including 
the dentist [6]. Because of this anxiety, many go without seeing the dentist on a regular basis, and thus may not have a dental home. Glassman and Miller [7] advocate for modalities that can complement or replace the pharmacological interventions provided by the CRNA as a method for achieving oral health for people with special needs. Understanding and addressing sensory processing and the sensory aspects of the dental visit may allow providers to implement strategies to improve the patient's cooperation and allow for better results [8]. According to literature from the past decade, animal assisted therapy (AAT) has been beneficial in addressing sensory impact in a variety of settings including, but not limited to, [9]. Documented benefits of AAT include improved physical, emotional, cognitive, and social functioning $[10,11]$; reduced blood pressure and triglyceride levels; and even reduced cardiovascular morbidity and mortality [12]. Two of the most downloaded journal articles for complementary therapies were related to AAT. One article was regarding pain in children [13] and the other focused on acute mental disorders in children [14].

The purpose of this project was to add an AAT team (a certified therapy dog and handler) to an interprofessional dental team and evaluate the impact of the AAT on the behaviors and anxiety levels of individuals with IDD. Specific focus was on using the therapy dog and handler to alter the environment of the dental office from one of lights, sounds and sensory stimulation from the dental office, to an environment of calming interaction on the individual with IDD

\section{METHODS}

\section{Design}

This project used at pre-test/post-test group design. The anxiety level and behaviors of individuals with IDD seen in the dental suite were assessed before the encounter with the AAT and following the encounter.

\section{Sample/Participants}

A convenience sample of thirty individuals with intellectual disabilities participated with the AAT enhanced interprofessional dental team. Participants were existing patients in the dental practice that had previously undergone a dental cleaning with sedation. The participants served as their own control. Participants were recruited for the study, and consent was obtained from family members or legal guardians prior to the scheduled study appointment. This minimized any disruption of typical office visit routines for the patients. Participants who required restorative treatments were excluded from the study. Patients' ages ranged from 24 to 66 years of age with an average age of 46 years. Males represented $50 \%$ of patients and females represented $50 \%$. Caucasians represented the largest group of patients at $56.7 \%$, African Americans at $36.7 \%$, Pacific Islanders at 3.3\%, and Hispanics at $3.3 \%$. Sixty percent of the patients had a profound level of disability. A majority (63.3\%) of these patients lived in-group homes with 7 or less residents. While only $23.3 \%$ or 7 of the patients had a physical disability, $90.0 \%$ had at least one chronic health condition in addition to their intellectual disabilities. Autism spectrum disorder was diagnosed in $33.3 \%$ of the patients and $26.7 \%$ of the population was also diagnosed with a neurologic impairment that included seizure disorders, cerebral palsy, and/or blindness.

\section{Setting}

The site for this project was a specialty sedation dental practice within a general dental practice located in the South Eastern region of the United States that provided care to individuals with intellectual disabilities. The practice had 250 patients with diagnoses primarily of autism spectrum disorder, Asperger's Syndrome, mental retardation, Down syndrome, psychiatric disorders, and Alzheimer's disease. The patients were from diverse cultural and socioeconomic backgrounds. An interprofessional team consisting of a dentist, a dental hygienist, a paramedic, and a CRNA provided care to this patient cohort one day per week. Care varied from routine hygiene visits to comprehensive restorative care.

\section{Data Collection}

Quantitative data was collected on anxiety using the Anxiety Depression and Mood Scale (ADAMS). This scale was developed to measure anxiety and mood changes in individuals of various intellectual levels. The subscale for anxiety was used for this study. The subscale consisted of seven behavioral items (nervous, does not settle down, tense, worried, anxious, panic attack, trembles) which cover five of the diagnostic criteria or symptoms of anxiety. Internal consistency(Cronbach's alpha) for the general anxiety subscale has been tested at 0.83 [15]. Scores range from 1-no anxiety, 2-minor anxiety, 3-mild anxiety, 4moderate anxiety, and 5-severe anxiety. The average score of the items were calculated in order to identify their level of anxiety with higher level indicating greater anxiety. 
A researcher-developed tool was used to measure the participant's behavior pre and post AAT intervention. The first of these items identified whether the patient was agitated, combative, pacing, or calm prior to the contact with the AAT and then after 10 minutes of contact with the AAT. These behaviors were measured as $0=$ behavior not present and $1=$ behavior present. Two additional items were used to assess whether the patient willingly went to the clinical area of the suite and whether they needed to be pre-medicated to go to the clinical area. This information was obtained from the chart regarding the patient's previous visit and compared to the response to the clinical area when accompanied by the AAT.

\section{Intervention}

Following approval by a university IRB, the CRNA recruited a certified therapy dog and handler to meet with individuals with IDD that had a scheduled appointment for routine dental care. Both the handler and dog were certified through Therapy Dog International. The handler volunteered his time for the study. The goal of using the certified therapy animal was to reduce the anxiety for the individuals with IDD prior to their dental exams and hygiene visits. Following written consent from the either the parent, caregiver, or legal guardian, the participants and their caregiver were escorted to a waiting area designed for this population. The CRNA collected baseline data using the ADAMS and the researcher developed tool. These tools were completed through an observation of the individual with IDD prior to receiving the AAT. Data was then collected after the individual with IDD had spent 10 minutes with the AAT. If the participant showed any signs of fear or aggression regarding the dog, the dog was immediately removed from the room. It should be noted that the dog intuitively remained a distance from anxious participants, but moved closely to calmer receptive participants allowing them to stroke or hold him. This timeline of 10-minutes was selected as it is the typical waiting time for patients in the dental office. The participant was then assisted to the dental suite accompanied the dog and hander. Once the IV was established and a state of anesthesia induced, the dog and handler exited the room.

\section{RESULTS}

Anxiety and behavioral changes of the individuals with IDD were assessed using descriptive and inferential statistics. The mean ADAMS score for the participants in this study was 4.30 (SD 0.79) out of a possible 5 prior to the introduction of AAT, which indicated moderate/severe anxiety. Following the AAT, the mean anxiety scores decreased to 2.93 (SD 0.86), which indicated a significant $(p<.01)$ reduction to $\mathrm{mild} /$ minor anxiety.

There were significant improvements in behaviors following the AAT using the Chi-Square $(p<.01)$. Behaviors were also assessed prior to introducing the AAT and then after 10 minutes of AAT. Prior to AAT, $83.3 \%$ of the participants exhibited agitation, 33.3\% exhibited combative behaviors, $53.3 \%$ paced and only $30 \%$ remained calm prior to entering the dental suite. When the AAT was introduced these numbers changed dramatically. Only $20 \%$ demonstrated agitation, 20 . \% were combative, and $22.2 \%$ paced, but most impressive $50 \%$ remained calm.

A chart audit was conducted of charts from the patient's previous visit in order to determine if the patient received pre-medication or required coaxing in order to proceed to the clinical area of the dental suite. Prior to the addition of the AAT, $83 \%$ of the study participants would not enter the dental suite without some form of pre-sedation medication or coaxing by staff. Following the initiation of AAT, only $16.7 \%$ of the participants refused to leave the waiting room when accompanied by the dog and handler. This difference was significant using the Chi-Square at $p<.01$.

\section{DISCUSSION}

This study aimed to investigate the outcomes of an interprofessional team's inclusion of AAT in a dental practice caring for individuals with IDD. Study results suggested that introduction of AAT resulted in an improvement in the patients' anxiety levels, cooperation improved, and there was a diminished need for presedation or anxiety medication for the study participants.

It should be noted that as the study progressed and participants returned for follow-up appointments, a noticeable increase in comfort and anticipation of the visit with the dog was apparent. A once terrifying office visit had blossomed into a partnership for better health between the patient, caregiver, and staff. Instead of the pacing and agitated behaviors patients were entering the office smiling and happy. Even more patients were agreeable to having the required monitors and intravenous fluids started. Leaving the caregiver was no longer a source of anxiety as the dog and handler accompanied them into the dental suite. 
The study has some limitations. First, this is a preliminary study with a small sample size and no control group. However, significant difference were found pre and post intervention suggesting that the incorporation of AAT on an interprofessional dental team that treats individuals with IDD can have a positive impact. A future study is now being conducted using a control group and a larger sample.

Based on the findings of this study, specialty practices that treat individuals with intellectual disabilities may consider adding AAT to their interprofessional team. This approach shows great potential in minimizing the anxiety and disruptive behaviors demonstrated with many individuals with IDD when in a healthcare setting.

\section{REFERENCES}

[1] Hermans $H$, Evenhuis $H M$. Factors associated with depression and anxiety in older adults with intellectual disabilities: results of the healthy aging and intellectual disabilities study. Int J Geriatr Psychiatry 2013; 28: 691-99. http://dx.doi.org/10.1002/gps.3872

[2] Boyle CA, Boulet SB, Schieve LA, Cohen RA, Blumberg SJ, Yeargin-Allsopp $M$, et al.Trends in prevalence of developmental disabilities in US children, 1997-2008. Pediatrics 2011; 127(6): 1034-42.

http://dx.doi.org/10.1542/peds.2010-2989

[3] Petersen PE. Global policy for improvement of oral health in the $21^{\text {st }}$ century-implications to oral health of World Health Assembly 2007, World Health Organization. Community Dent Oral Epidemiol 2009; 37(1): 1-85.

http://dx.doi.org/10.1111/j.1600-0528.2008.00448.x

[4] US Department of Health and Human Services. Oral health in America: report of the Surgeon general--Executive Summary. Rockville, MD: National Institute of Dental and Craniofacial Research, National Institute of Health. 2000. [cited 2015 Nov 18]. Available from: http://www.nidcr.nih.gov/ DataStatistics/SurgeonGeneral/Report/ExecutiveSummary.ht $\mathrm{m}$

[5] Cantlay K, Williamson S, Hawkings J. Anesthesia for dentistry continuing education in anesthesia. Contin Educ Anaesth Crit Care Pain 2005; 5(3): 71-5.

http://dx.doi.org/10.1093/bjaceaccp/mki020
[6] Collado V, Faulks D, Hennequin R. A survey of the difficulties encountered during routine hygiene and health care by persons with special needs. Disabil Rehabil 2008; 30(14): 1047-54.

http://dx.doi.org/10.1080/09638280701616574

[7] Glassman P. A review of guidelines for sedation anesthesia, and alternative interventions for people with special needs. Spec Care Dentist 2009; 29(1): 9-16. http://dx.doi.org/10.1111/j.1754-4505.2008.00056.x

[8] Kuhaneck HM, Chisholm EC. Improving dental visits for individuals with autism spectrum disorder through an understanding of sensory processing. Spec Care Dentist 2012; 32(6): 229-33.

http://dx.doi.org/10.1111/j.1754-4505.2012.00283.x

[9] Coakley AB, Mahoney EK. Creating a therapeutic and healing environment with a pet therapy program. Complement Ther Clin Pract 2009; 15(3): 141-6. http://dx.doi.org/10.1016/j.ctcp.2009.05.004

[10] Nepps P, Stewart C, Bruckno SR. Animal-assisted activity: effects of a complementary intervention program on psychological and physiological variables. J Evid Based Complement Altern Med 2014; 19(3): 211-16. http://dx.doi.org/10.1177/2156587214533570

[11] Esbensen AJ, Rojahn J, Aman MB, Rudrich S. Reliability and validity of an assessment instrument for anxiety, depression, and mood among individuals with mental retardation. J Autism Dev Disord 2003; 33(6): 617-29. http://dx.doi.org/10.1023/B:JADD.0000005999.27178.55

[12] Cole KM, Gawlinski A, Steers N, Kotlerman J. Animalassisted therapy in patients hospitalized with heart failure 2007; 16(6): 574-85.

[13] Braun C, Stangler T, Narveson J, Pettingell S. Animalassisted therapy as a pain relief intervention for children. Complement Ther Clin Pract 2009; 15(2): 105-9. http://dx.doi.org/10.1016/j.ctcp.2009.02.008

[14] Stafanini MC, Martina A, Allori P, Galeotti F, Tani F. The use of animal-assisted therapy in adolescents with acute mental disorders: a randomized controlled trial. Complement Ther Clin Pract 2015; 21(1): 42-6. http://dx.doi.org/10.1016/i.ctcp.2015.01.001

[15] Esbensen AJ, Rojahn J, Aman MG, Ruedrich S. Reliability and validity of an assessment Instrument for anxiety, depression, and mood among individuals with mental retardatation. J Autism Dev Disord 2003; 33(6): 617-29. http://dx.doi.org/10.1023/B:JADD.0000005999.27178.55 